\title{
Acute disseminated encephalomyelitis due to Rickettsia conorii infection
}

\author{
D Woolf, ${ }^{1}$ MB ChB, FCP (SA); M Jordaan, ${ }^{2}$ MB ChB, FRCR (London); G Maartens, ${ }^{1}$ MB ChB, MMed (Med) \\ ${ }^{1}$ Department of Medicine, Faculty of Health Sciences, University of Cape Town, South Africa \\ ${ }^{2}$ Morton and Partners, Cape Town, South Africa
}

Corresponding author: G Maartens (gary.maartens@uct.ac.za)

Acute disseminated encephalomyelitis (ADEM) is an immune-mediated acute inflammatory demyelinating disorder, which typically occurs after viral infections or immunisation. We present a case of a man with acute Rickettsia conorii infection whose diagnosis was delayed. He presented with fever, headache, an eschar and an acute paraplegia. The R. conorii IgM serum titre was 1:128. Magnetic resonance imaging showed multifocal lesions in the brain and spinal cord consistent with inflammatory demyelination. The patient responded well to doxycycline and a short course of high-dose corticosteroids. To our knowledge this is the first case of ADEM associated with Mediterranean spotted fever - we found a previous report of ADEM in a child with Rocky Mountain spotted fever, whose diagnosis of rickettsial infection was also delayed. We hypothesise that delayed diagnosis of spotted fever group rickettsial infections could rarely result in ADEM.

S Afr Med J 2021;111(4):307-308. https://doi.org/10.7196/SAMJ.2021.v111i4.15308

\section{Case presentation}

A 48-year-old man was admitted via the emergency unit to the medical ward with fever, headache and an acute paraplegia. He was previously well. Four weeks prior to admission he hiked in rural Mpumalanga Province and in the Kruger National Park - a game reserve in South Africa (SA). He then travelled to southern Mozambique. He was taking atovaquone-proguanil as malaria prophylaxis. Two weeks before admission, while still in Mozambique, he noted a pustular lesion on his left lower abdomen, which subsequently developed a black centre. He developed tender swollen glands in his left groin, severe headache, fever and rigours, malaise and myalgia. He did not develop a rash. Two days after the onset of his illness, he returned to his home in Cape Town, SA and consulted his general practitioner, who prescribed a 5-day course of cefpodoxime for lymphadenitis. His symptoms did not improve, and the general practitioner then prescribed amoxicillin-clavulanate, which also had no effect on his symptoms. He developed thoracic back pain radiating anteriorly, with reduced sensation below this level, and progressive weakness of his legs over 2 days. He again consulted his general practitioner, who started doxycycline for presumed rickettsial infection. He was admitted the next day (14 days after the onset of his illness) with worsening leg weakness, resulting in an inability to walk without support.

On admission, he was sweaty, with a temperature of $37.8^{\circ} \mathrm{C}$. His blood pressure was normal and pulse was $100 \mathrm{bpm}$. An eschar was present on the left lower abdomen. He had multiple small, tender, enlarged left inguinal lymph nodes. There was no rash. The patient had a tender enlarged bladder, which drained $1.5 \mathrm{~L}$ on catheterisation. There was no meningism. He had a spastic paraplegia with moderate (grade 3/5) weakness. Sensation was diminished in the legs and trunk, with a sensory level at T5. Fundoscopy was normal. Mild confusion was present.

A complete blood count showed mild neutrophilia. Transaminases were minimally elevated. Magnetic resonance imaging (MRI) of the spinal cord (Fig. 1 (A and B)) showed a lesion in the mid- thoracic region, with surrounding oedema, and enhancement after administration of gadolinium; there was no cord compression. MRI of the brain, with contrast enhancement, showed multifocal white-matter lesions affecting the juxtacortical $\mathrm{U}$-fibres, the corpus callosum and the brachium pontis (Fig. 2 (A and B)). The MRI features were highly suggestive of an inflammatory demyelinating process. Cerebrospinal fluid (CSF) analysis showed normal glucose, protein $1.53 \mathrm{~g} / \mathrm{L}$, lymphocytes $542 \times 10^{9} / \mathrm{L}$ and neutrophils $6 \times 10^{9} / \mathrm{L}$; bacterial and fungal cultures were negative, cryptococcal antigen was absent and oligoclonal bands were not detected. Rickettsia conorii IgM serology was positive in the serum at a titre of 1:128, but polymerase chain reaction (PCR) for rickettsial DNA was negative.

Doxycycline $100 \mathrm{mg}$ 12-hourly was continued to complete a 7-day course. A diagnosis of acute disseminated encephalomyelitis (ADEM) was made, for which the patient was administered a 5-day course of high-dose corticosteroids. The headache resolved rapidly. The patient was discharged after 8 days, when he had normal power, could walk unassisted and bladder function was normal. He had persistent thoracic radicular pain, for which he was given pregabalin, with partial relief. On follow-up 12 weeks later, the radicular pain persisted but was improving. A repeat MRI of the brain and spinal cord showed marked reduction in the size and number of lesions.

\section{Discussion}

Our patient presented with classic clinical features of $R$. conorii infection: an eschar, regional lymphadenitis, fever and severe headache, confirmed by a strongly positive IgM serological test. Mediterranean spotted fever (MSF) is common in the areas where he travelled before his symptoms began. ${ }^{[1]}$ The diagnosis of MSF was delayed; he then developed a paraplegia with multiple inflammatory demyelinating lesions in the brain and spinal cord, as detected on MRI. The neurological symptoms and MRI features improved over a few months, with no new neurological or MRI features for 3 months. The neurological symptoms and MRI features are consistent with ADEM ${ }^{[2]}$ Encephalopathy, which was mild in our patient, occurs 

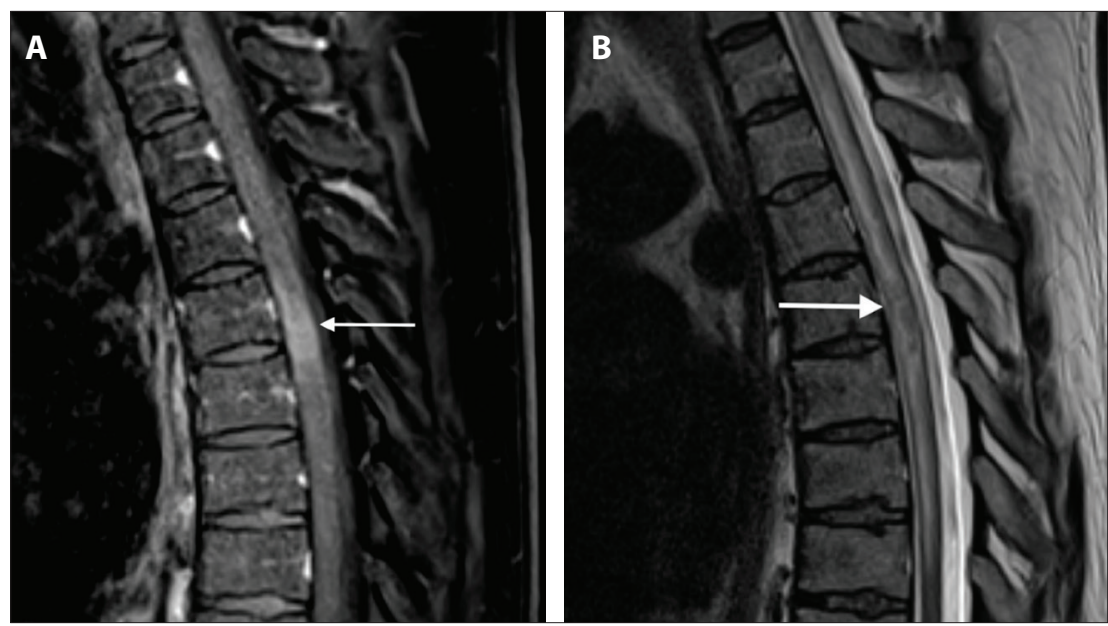

Fig. 1. Magnetic resonance imaging of the spinal cord on admission. (A) Focus of signal alteration in the mid-thoracic cord on the T2-weighted image (arrow), with surrounding oedema. (B) Post-contrast enhancement on T1-weighted imaging after gadolinium administration (arrow).
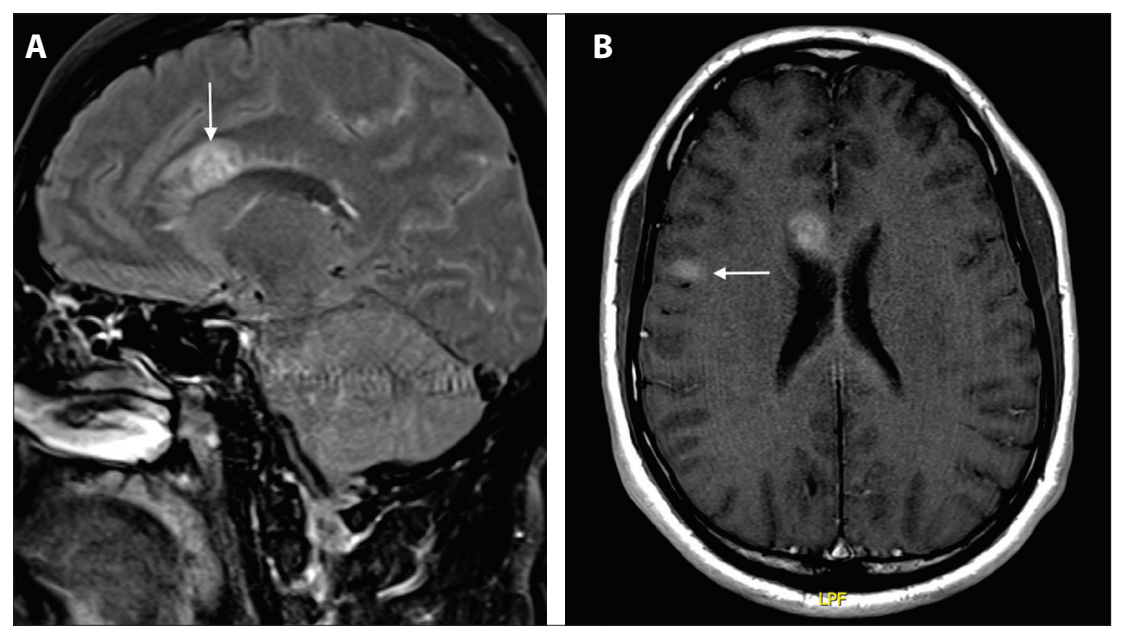

Fig. 2. Magnetic resonance imaging of the brain on admission. (A) Sagittal midline FLAIR (fluidattenuated inversion recovery) image demonstrates multiple, linear, radially orientated foci of signal abnormality in the corpus callosum. The dominant focus is situated in the anterior callosal body (arrow). (B) Axial T1-weighted image obtained after gadolinium contrast use. The dominant lesion in the corpus callosum and another in the juxtacortical right frontal white matter (arrow) demonstrate post-contrast enhancement.

more commonly in children than in adults. ${ }^{[3]}$ MRI scans demonstrate that the spinal cord is involved in about a third of adults with ADEM. ${ }^{[3]}$ The active CSF in our patient is an atypical feature in ADEM (typically leukocytes $<50 \times 10^{9} / \mathrm{L}$ and protein $\left.<1 \mathrm{~g} / \mathrm{L}\right)$; ${ }^{[4]}$ we attributed his CSF abnormalities to rickettsial infection.

MSF often has neurological manifestations. Severe headache with aseptic meningitis, as in our patient, is common, but overt encephalitis is rare. ${ }^{[5]}$ MSF rarely causes cerebral vasculitis, which can result in small infarctions. ${ }^{[6]}$ The MRI appearances in our patient were not consistent with vasculitis, but were consistent with $\mathrm{ADEM}^{[2]}$ We were unable to find any reports of ADEM complicating MSF. We are aware of only one other case of ADEM due to rickettsial infection - in a 7-year-old boy with Rocky Mountain spotted fever: ${ }^{[7]}$ the latter diagnosis was delayed, as in our case, with leukocytes $>50 \times 10^{9} / \mathrm{L}$ in the CSF. We hypothesise that delayed diagnosis of spotted fever group rickettsial infections could rarely result in ADEM.

ADEM is an immune-mediated postinfectious or post-immunisation inflammatory demyelinating illness, mainly caused by viruses. ADEM is usually a monophasic illness, as in our patient. It is important to recognise $\mathrm{ADEM}$, as it is steroid responsive, although there are no randomised controlled trials to support the use of

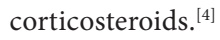

Our patient could have had African tick bite fever, which is also prevalent in the areas where he travelled ${ }^{[1]} R$. africae cross-reacts serologically with $R$. conorii. ${ }^{[9]}$ Culture or PCR of blood or an eschar biopsy is required for the definitive diagnosis of $R$. africae, ${ }^{[9]}$ but in our patient the PCR was negative in the blood.

In conclusion, we have described a case of ADEM in a patient with MSF who responded well to treatment with doxycycline and highdose corticosteroids. It is possible that the delayed diagnosis, which was also a feature in the published case report of a child with Rocky Mountain spotted fever (the only other report of ADEM associated with a rickettsial infection we could find), was a factor in the development of ADEM.

\section{Declaration. None.}

Acknowledgements. None.

Author contributions. DW provided the clinical data; MJ interpreted the MRI scan; and GM supervised the study and wrote the first draft. All authors approved the final version.

Funding. None.

Conflicts of interest. None.

Parola P, Paddock CD, Socolovschi C, et al. Update on tickborne rickettsioses around the world: A geographic approach. Clin Microbiol Rev 2013;26(4):657-702. https://doi.org/10.1128/ CMR.00032-13

2. Marin SE, Callen DJ. The magnetic resonance imaging appearance of monophasic acute disseminated encephalomyelitis: An update post application of the 2007 consensus criteria. Neuroimaging Clin N Am 2013;23(2):245-266. https://doi. Neuroimaging Clin N Am
org/10.1016/j.nic.2012.12.005

3. Koelman DL, Chahin S, Mar SS, et al. Acute disseminated encephalomyelitis in 228 patients: A retrospective, multicenter US study. Neurology 2016;86(22):2085-2093. https://doi.org/ 10.1212/WNL.0000000000002723

4. Pohl D, Alper G, van Haren K, et al. Acute disseminated encephalomyelitis: Updates on an inflammatory CNS syndrome. Neurology 2016;87(9 Suppl 2):S38-S45. https://doi.org/10.1212/ WNL.0000000000002825

5. Aliaga L, Sánchez-Blázquez P, Rodríguez-Granger J, Sampedro A, Orozco M, Pastor J. Mediterranean spotted fever with encephalitis. J Med Microbiol 2009;58:521-525. https://doi, org/10.1099/jmm.0.004465-0

6. Brinar VV, Habek M. Rare infections mimicking MS. Clin Neurol Neurosurg 2010;112(7):625-628. https://doi.org/ 10.1016/j.clineuro.2010.04.011

Wei TY, Baumann RJ. Acute disseminated encephalomyelitis after Rocky Mountain spotted fever. Pediatr Neurol 1999(1);21:503-505. https://doi.org/10.1016/s0887-8994(99)00028-4

8. Roch N, Epaulard O, Pelloux I, et al. African tick bite fever in elderly patients: 8 cases in French tourists returning from South Africa. Clin Infect Dis 2008;47(3):e28-e35. https://doi. org/10.1086/589868

9. Jensenius M, Fournier PE, Kelly P, Myrvang B, Raoult D. African tick bite fever. Lancet Infect Dis 2003;3(9):557-564. https://doi. org/10.1016/s1473-3099(03)00739-4

Accepted 1 December 2020. 